\title{
MUJER-RESISTENCIA COMO PROYECTO CONTRAIMPERIAL: PROPUESTA DESDE UNA SEMIÓTICA INTERCULTURAL
}

\author{
LIBERTINE POETESS IN AL-ANDALUS
}

\author{
Rodrigo Browne \\ Universidad Austral, Valdivia, Chile
}

\section{Resumen:}

Los discursos teóricos sobre la mujer se detienen, generalmente, en lecturas cerradas, homogéneas, donde, a pesar de las categóricas posturas feministas, la figura masculina es el punto de debate o el trámite en cuestión para romper los estereotipos en torno al mal llamado sexo débil. Nuestra propuesta, en síntesis, tratará de resistir a las lecturas binarias, y pretenderá potenciar una semiótica intercultural que indagará en las escrituras rizomáticas que, desde los postulados de Gilles Deleuze y Félix Guattari (1976), se enfrenten a los proyectos heredados de la modernidad y que, en terrenos posmodernos (del capitalismo tardío), se reconocen bajo la denominación del Imperio. Por lo mismo planteamos esta mujer-resistencia como una resistencia contraimperial, como una vía de escape que, en palabras del propio Deleuze (1977) permita: “(...) llegar a tartamudear en su propia lengua (...) Ser como un extranjero en su propia lengua. Trazar una línea de fuga".

\section{Palabras claves:}

Mujer, Imperio, cultura.

\begin{abstract}
:
The theoretical discourses about woman fix themselves, generally, in enclosed homogeneous readings; where, notwithstanding the categorical feminist positions, the male image is the point of debate or the affair in quarrel in order to break through the stereotypes related to the ill named weak sex. Our proposal, to sum up, will intend to resist the binary readings, but will aim to empower an intercultural semiotics that will investigate in the rizomatic writings which, departing from the ideas of Gilles Deleuze y Félix Guattari (1976), will face the projects inherited by modernity and which, in postmodern grounds (of the late capitalism), recognize themselves under the name of counterimperial, as a way out that, in Deleuze (1977) words will allow us to: "stutter in our own tongue (...) to be like a foreigner in our own tongue. Trace an elopement line".
\end{abstract}

\section{KEY WORD:}

Woman, Empire, culture. 
Ningún poder falocrático resiste a Cicciolina.

Ningún poder político resiste al terrorismo.

Son cánceres, pues aplican el paso de la metáfora a la metástasis

J. Ibáñez

I. Los discursos teóricos sobre la mujer se detienen, generalmente, en lecturas cerradas, homogéneas, donde, a pesar de las categóricas posturas feministas ${ }^{1}$, la figura masculina es el punto de debate o el trámite en cuestión para romper los estereotipos en torno al mal llamado "sexo débil". La mujer, aunque motor de investigaciones, termina, como de costumbre, sumida en un discurso binario (macho-hembra) auspiciado por una sociedad como la nuestra que, desde la modernidad, estimula (y cuestiona) el crecimiento de las ciencias humanas (Foucault, 1966, 1986), “(...) prevé que los discursos de las mujeres 'se dicen' y pasan en el mismo acto en que han sido pronunciados mientras que los discursos de los hombres son discursos fundadores..." (Arriaga, 1998).

Así también lo entiende Jesús Ibáñez, al indicar que la razón masculina es una razón negativa que organiza prohibiendo. Prohibiendo la relación reflexiva (masturbación), la relación simétrica (amistad), las relaciones transitivas inmediatas, por una parte, de semejanza (homosexualidad) o, por otra, de contigüidad (incesto): “La dominación de las mujeres por los hombres es la matriz de todas las dominaciones: la primera y la más intensa. La mujer es el primer objeto producido. Y la producción es una actividad masculina" (Ibáñez, 1994: 64-65).

Al respecto, Rafael Vidal explica que los movimientos feministas se enmarcan en una permanente descomposición “(...) del orden simbólico ilustrado..." (Vidal, 2001: 1) de influencia occidental y, siguiendo las políticas que Vázquez Medel (1999) desarrolla en este ámbito, androcéntrico. Es necesario, en consecuencia, des-emplazar (re-emplazar) a la mujer, es decir, lo femenino debe renunciar a su alteridad masculina (Vidal, 2001).

Acorde con este proceso de des-emplazamiento (re-emplazamiento), Hélène Cixous aboga, desde una mirada posmoderna, por una escritura que supere los agonizantes residuos falocéntricos y, producto de esta desarticulación, incite a participar de un proyecto que se manifieste "(...) entre-ella y yo y tú entre el yo donde uno es siempre infinitamente más de uno y más que yo..." (Cixous, 1995: 65). Mirada trasgresora que no teme alcanzar un límite, sino que goza de la carencia de control: “iNunca llegaremos al final! Ella pasa por los amores defensivos, las maternidades y devoraciones" (Ibidem), luchando por un espacio hiperactivo, inestable, abierto, transicional que desautorice -quitar autoría, “(...) ¿qué importa quién está hablando?” (Foucault, 1969: 1)- las reglas

1 "La rebelión masculina es luciferina. Lucifer dijo: no serviré (a nadie, para nada). Es un desafío frontal al poder: le costó el infierno. Muchas feministas siguen la vía de Lucifer: arriesgan el mismo destino" (Ibáñez, 1994: 65) que instalan a la mujer en las nocturnas coordenadas del "continente negro", siempre perfectamente bien ordenado por la pureza e incontaminación de lo "blanco" (Browne, 2002).

II. Pero la crítica no debe, necesariamente, cerrarse a las cuestiones y consecuencias de la modernidad, sino debe detenerse y analizar las acciones que en el campo de la posmodernidad han surgido en torno a estos temas. En este contexto, entendemos las propuestas de Cixous. El complejo debate modernidad-posmodernidad ha sido hábilmente analizado por muchos pensadores (Lyotard, Vattimo, Jameson, Hottois, Virilio, García Canclini, Lanceros, Vázquez Medel, Talens, Fernández Serrato, Subirats) que han propuesto diversas lecturas a favor y en contra de dichos conceptos. Para nosotros, uno de los textos claves en relación a esta disyuntiva teórica y sus consecuencias es Imperio (2000) de Michael Hardt y Antonio Negri. En relación a esta obra, Víctor Silva Echeto (2003a) señala que el estudio de Hardt y Negri ubica a la posmodernidad en tránsito hacia un nuevo modelo, “(...) una lógica y una estructura de dominio nuevas: en suma una nueva forma de soberanía" (Hardt y Negri, 2000, 2002: 11) que ellos mismos denominan imperio².

Para ambos teóricos las formas modernas de poder que los críticos posmodernos y poscoloniales se preocupan por describir y combatir no ejercen ya tanta influencia en nuestra sociedad, así, consideran que desconocen el actual poder del Imperio que ya no es colonial, ni se ampara en la Ilustración (Silva Echeto, 2003a: 6).

Podemos, en tanto, desprender de la interpretación que Silva Echeto hace sobre la propuesta de Hardt y Negri que éstos teóricos superan la noción de imperialismo, como paradigma de la modernidad, y dan paso a un periodo posmoderno imperial. En síntesis, pretenden estudiar el nuevo orden político-social-económico de nuestros tiempos globalizados o, considerando el neologismo de Paul Virilio (1995), glocalizados y su vinculación con el tránsito que anuncia Foucault (1975) y diagnostica Deleuze (1993) entre sociedades disciplinarias y sociedades de control ${ }^{3}$.

Además, Hardt y Negri sostienen que la disciplina surge como una dominación patriarcal falocéntrica que se erige por medio de una trama de terminales que crean e imponen las normas de una sociedad determinada. Las sociedades de control, en cambio, son resultado de las últimas maniobras de la modernidad y estimulan el nacimiento de la posmodernidad, quedando el poder en manos de nuevas máquinas

2 Hardt y Negri explican que los teóricos posmodernos se refieren al fin de la soberanía moderna y presentan soluciones frente a las divisiones binarias, con el propósito de proponer un pensamiento de pluralidad y multiplicidad. "Por más que lo hagan de manera confusa o inconsciente, éstos teóricos indican el tránsito hacia la constitución del imperio" (Hardt y Negri, 2000, 2002: 134).

3 Mayor información sobre este tema en particular se puede encontrar en BROWNE, Rodrigo (2002):"Panóptico", "espectáculo" y "control": observaciones para una propuesta iconofágica. Congreso "Comunicación y Desarrollo en la sociedad global de la información". III Encuentro Iberoamericano de Economía Política de la Comunicación, 17, 18 y 19 de julio, Facultad de Comunicación. Universidad de Sevilla. España 
que perturban derechamente a los cerebros por medio de sistemas de comunicación, redes informativas y a los cuerpos a través de las asistencias sociales y el control de sus actividades y procedimientos, “(...) la sociedad de control podría caracterizarse por una intensificación y una generalización de los aparatos normalizadores del poder disciplinario que animan internamente nuestras prácticas comunes y cotidianas..." (Hardt y Negri, 2000, 2002: 36).

Con esto, se derriba uno de los principales edificios de la modernidad como es el estado-nación, rompiéndose las estructuras binarias y creando un nuevo espacio de acción más libre, "democrático" y en el cual se escuchan las voces de la diferencia pero, a su vez, se abre un nuevo modelo de control que acepta la diferencia y se ubica en los deslindes de los discursos dicotómicos. A diferencia de la soberanía imperialista, la soberanía imperial se disemina por los márgenes, en la elasticidad de las fronteras y las identidades.

El tránsito al imperio se da a partir del ocaso de la soberanía moderna. En contraste con el imperialismo, el imperio no establece ningún centro de poder y no se sustenta en fronteras o barreras fijas. Es un aparato descentrado y desterritorializador de dominio que progresivamente incorpora la totalidad del terreno global dentro de sus fronteras abiertas y en permanente expansión. El imperio maneja identidades híbridas, jerarquías flexibles e intercambios plurales a través de redes adaptables de mando. Los colores nacionales distintivos del mapa imperialista del mundo se han fusionado y mezclado en el arco iris global imperial (Hardt y Negri, 2000, 2002: 12).

El peso del imperio se reparte rápidamente por todos los escondrijos del orden social y nunca se detiene en un lugar delimitado, logrando, a partir de tal virtud, concebir y delinear los espacios por los cuales circula y de los cuales se alimenta. El imperio logra administrar y controlar la vida social. La idea no consiste, como en épocas anteriores, en dejarse regir por un centro unitario de operaciones, cuya labor era abandonar en el afuera todo lo que se alejara de sus principios y planteamientos. El imperio busca intervenir los discursos binarios, asumiendo las alternativas terceroespaciales (Bhabha, 1994) y abriendo el juego de la diferencia, de los antiesencialismos y de las proyecciones híbridas, mestizas y criollas. Es decir, incluyendo y dispersando al Otro eternamente excluido. Estas maniobras imperiales son las que hay que resistir. Resistencia que pretendemos estimular en el amplio ámbito de la misma posmodernidad para que, así, podamos, desde una postura mujer-resistente, releer los vicios que la nueva soberanía ha inculcado. Nuestro propósito es deconstruir las visiones tercero-espaciales culturales que fueron acomodadas en beneficio del imperio posmoderno.

III. Dicha estrategia de resistencia debe ser provocadora y transgresora. Debe romper con las figuras impuestas por los modelos imperantes desde el Renacimiento hasta nuestros días ${ }^{4}$. La resistencia para confrontar al imperio y su mercado globalizado debe plantearse igualmente desde un punto de vista mundializado, “No es posible oponer resistencia al imperio a través de un proyecto que apunte a lograr una autonomía limitada, local (...) debemos atravesar el imperio y salir del otro lado" (Hardt y Negri, 2000, 2002: 186). Es necesario proponer un ejercicio de contraglobalización o, más bien contraimperial.

En este tránsito de la modernidad a la posmodernidad, ¿existe un lugar desde donde podamos lanzar nuestra crítica y construir una alternativa? $\mathrm{O}$, si estamos confiados al no lugar del imperio, ¿podemos construir un potente no lugar y llegar a concretarlo como el terreno propicio para crear un republicanismo posmoderno? (Hardt y Negri, 2000, 2002: 187)

El proyecto contraimperial, si continuamos con la nomenclatura de Hardt y Negri, podemos asociarlo con la fuerza activa y rebelde que debe ejercer la mujer en este ejercicio de resistencia al nuevo orden. Dicha rebelión mujer-resistencia no debe caer en los juegos controladores que la misma posmodernidad pone sobre el tapete. Ibáñez precisa, al respecto, que entre estos modelos existen varios tipos de feminismos dignos de ser cuestionados. En principio, un feminismo converso: “(...) el de las mujeres que quieren ser iguales a los hombres -como las del PSOE- (acceder al numerador de la razón)" (Ibáñez, 1994: 66). También, argumenta este investigador, hay un feminismo perverso: “(...) el de las mujeres que quieren dar la vuelta a la tortilla -como las reivindicativas del MC- (invertir el numerador y el denominador)" (Ibidem). En tercer lugar, continúa Ibáñez, se presenta un feminismo subversivo: “(...) el de las mujeres que quieren abolir la dominación -como las anarquistas- (borrar la barra que separa el numerador del denominador)" (Ibidem). Pero, además y este es el punto que nos interesa para la resistencia contraimperial, existe un feminismo reversivo: “(...) el de las mujeres que hacen girar esa barra hasta hacerla estallar" (Ibidem).

Para Ibáñez, el último de estos feminismos es el único que tiene la capacidad de seducir y explica que los otros son sólo productivos, ya que intentan revalorizar a la mujer. La idea, sostiene el teórico, es “(...) desafiar a los machos a ser más machos”

4 No hay que olvidar, frente al combate para con la Ilustración, que los esfuerzos de la posmodernidad se centran en lo que Hardt y Negri llamaron la segunda modernidad. Estos teóricos anunciaron que la modernidad no es un fenómeno único y homogéneo, sino un proceso pleno de contradicciones y conflictos, detectando la existencia de, por lo menos, dos modernidades. "La primera es la iniciada por la revolución del humanismo del Renacimiento (...) con el descubrimiento del lugar de inmanencia y el elogio de la singularidad y la diferencia" (Hardt y Negri, 2000, 2002: 131) y la segunda tiene la intención de instalar la fórmula precisa para contener las fuerzas utópicas de la primera modernidad a través de la construcción de dualismos y, para culminar sus propósitos, dar vida a la noción de soberanía moderna. La posmodernidad “(...) debería aclararse que en realidad están atacando a la segunda tradición de nuestro esquema (y, desafortunadamente, ignorando o eclipsando la primera)" (Ibidem), es decir, las teorías posmodernas no se enfrentan estrictamente a los modelos de la Ilustración, sino que a la conformación del Estado-nación soberano como segunda etapa de la modernidad. 
(Ibidem) porque la estrategia de la producción es el deseo $^{5}$, en cambio el de la seducción es el desafío.

La rebelión seductora es un sobresometimiento a ese poder. Las rebeliones frontales refuerzan el poder: la conversa (que suplica al poder que sea menos poder) lo reforma la perversa (que intenta que el poder sea otro poder) lo invierte, la subversiva (que exige al poder que no sea poder) lo revoluciona. La reversiva (que desafía al poder a que sea más poder) pone al poder en una tesitura imposible: pues obliga a exacerbarse hasta extinguir la relación por exterminio de los términos (Ibáñez, 1994: 66).

La radicalización de la alternativa propuesta por Ibáñez podemos llegar a observarla en los estudios que Gilles Deleuze y Félix Guattari realizan en torno al mito de Edipo (El AntiEdipo. Capitalismo y esquizofrenia, 1972, 1998). Desde este punto de vista y, para nosotros, desde los postulados recientemente desarrollados, es loable que la mujer contenga tanto hombres como el mismo hombre y el hombre, por su parte, mujeres capaces de entrar unos en otros, unos con otros, interviniendo el orden normativo de los sexos. "Hacer el amor no se reduce a hacer uno, ni siquiera dos, sino hacer cien mil (...) no uno ni siquiera dos sexos, sino n... sexos" (Deleuze y Guattari, 1972, 1998: 305).

IV. Acto seguido, consideramos pertinente estimular un giro trans einterdisciplinario que nos permitirá continuar fundamentando la propuesta contraimperial que tratamos de elucidar. Esta postura desde lo político-social que manejamos con, por ejemplo, Hardt y Negri e Ibáñez la conjugamos con algunas proyecciones interculturales que, basada en una semiótica plural y heterogénea, nos incita a habilitar un activo juego semiótico-intercultural. Para Miquel Rodrigo Alsina (2000) existe un clima intelectual, un cambio epistemológico, favorable para desarrollar posturas que se aproximen a una semiótica intercultural. Iniciativa que, siguiendo a Silva Echeto, entiende el fenómeno intercultural como una cuestión cercana a las estrategias entre que derivan, en diferentes momentos, de Jacques Derrida y Gilles Deleuze: “El entre conjura toda propuesta de la identidad como unidad, cualquier posibilidad de transformar esenciales a identidades movibles, que son modulables como masas plásticas que cambian permanentemente" (Silva Echeto, 2003b: 148)

Por eso es necesario activar una semiótica que se deslice entre los factores que tratan de imponer un orden del discurso: posición que se aproxima a definiciones o identidades cerradas que pretenden explicarlo todo, sin escuchar otras voces.

5 En torno al deseo y a la disposición del mismo, consideramos pertinente recalcar la presente aclaración que hace Gilles Deleuze. Dicho pensador francés explica que el poder no es quien dispone, sino los agenciamiento de deseo quienes habilitarían, siguiendo una de sus dimensiones, los adiestramientos de poder. El poder puede ser deseado porque el primero de éstos es un apéndice del segundo y este último es, por ende, el componente de un micro-análisis. Existe una superioridad del deseo sobre el poder y el perfil secundario que éste posee, “(...) sus operaciones siguen teniendo un efecto represivo, ya que no aplastan el deseo como dato natural, sino los puntos de disposición del deseo" (Deleuze, 1977, 1995: 15).
En consecuencia, nuestra intención no es detenernos en las puntualidades clásicas de la semiología positivista -"Pero el par saussuriano lengua/habla -como todas las oposiciones que construyen los estructuralistas- es (...) estático" (Ibáñez, 1994: 39)-, sino recuperar las nociones más transgresoras que en este sentido encontramos en una semiótica que tiende a cuestionar la soberanía, no sólo del imperialismo de antaño, sino también del imperio posmoderno. En este sentido, Rodrigo Alsina ofrece algunos pasos cruciales para, desde nuestro punto de vista, dessemiotizar la ortodoxa semiótica y abrir interesantes y renovadas opciones a la hora de traducir y/o interpretar un texto específico.

En la actualidad se empiezan a transgredir los límites, las disciplinas descubren que sus fronteras son blandas y que sus objetos de estudio no son de su exclusiva propiedad. En este contexto la semiótica puede sentirse muy a su aire porque, como apunta Urrutia (2000: 82), “La semiótica no corresponde (...) a lo que suele considerarse una disciplina escolar (...) Por su propia naturaleza es interdisciplinar, extradisciplinar o, me gusta más, indisciplinada..." (Rodrigo Alsina, 2000: 1).

Indisciplinamiento o, en este caso particular, dessemiotización que puede llegar, no sólo a la anulación del significado dentro del ejercicio semiótico-deconstructivo que entendemos de Derrida, sino también a una puesta en duda, en ocasiones, del propio significante que, desde los estudios deleuzianos, nos hace reflexionar sobre un asignificante. $\mathrm{Al}$ respecto, en una primera etapa, Deleuze revela una paradoja en forma de antinomia: “(...) dadas dos series, una significante y otra significada, una presenta un exceso y otra un defecto, por los cuales se remite una a otra en eterno desequilibrio" (Deleuze, 1969, 1989: 69) e indica que ambas series heterogéneas (una significante y otra significada) se inclinan hacia un diferenciante. Dicho diferenciante figura como un estimulador de singularidades que circula entre las dos series: "Además tiene la propiedad de estar desplazado siempre respecto de sí mismo, de 'faltar a su propio lugar', a su propia identidad, a su propia semejanza, a su propio equilibrio" (Ibidem, 70) Aunque los últimos argumentos deleuzianos nos permiten poner en jaque la posición semiótica tradicional, este intelectual, tiempo después, transgrede un tanto más su postura. En conjunto con Félix Guattari y, probablemente, influenciado por los análisis de este último pensador francés, Deleuze supera la misma figura significado-significante que estimula al diferenciante como tercero y desautoriza al mismo significante. Es aquí donde brota -proveniente, sin duda, de la no-arborescencia rizomática- el no significante o asignificante que se escapa del carácter lingüístico al que remite el propio significante, dentro de la semiótica estructuralista. En palabras de Guattari:

No tenemos nada que ver con el significante (...) La oscuridad de nuestra crítica del significante se debe a que se trata de una entidad difusa que todo lo reduce a una máquina obsoleta de escritura. La oposición exclusiva y coercitiva entre significante y 
significado está obsesionada pro el imperialismo del Significante, tal y como emerge con las máquinas de escritura (...) Nuestra hipótesis es esta: el Significante es el signo del gran Déspota que, al retirarse, libera una región que puede descomponerse en elementos mínimos entre los que existen relaciones regladas. Esta hipótesis tiene la ventaja de explicar el carácter tiránico, terrorista y castrador del significante. Se trata de un enorme arcaísmo que remite a los grandes imperios (Guattari, 1992, 1996: 39).

V. Por lo mismo, este ejercicio contraimperial -que planteamos desde una tendencia (des)semiótica intercultural- nos invita a reflexionar sobre el empírico proyecto mujer-resistencia que se activa como una trasgresión contraimperial, como una vía de escape que, según el propio Deleuze, puede: “(...) llegar a tartamudear en su propia lengua (...) Ser como un extranjero en su propia lengua. Trazar una línea de fuga" (Deleuze, 1977, 1997: 8) o, en palabras de Cixous, “(...) escribía a nadie, a todas, a la escritura, en una lengua extranjera, no la hablo, pero mi corazón la comprende, y sus palabras silenciosas en todas las venas de mi vida se han traducido en sangre demente, en sangre-alegría" (Cixous, 1979, 1995: 110). Líneas de fuga que consientan sumergirse en las rendijas, escaparse de la dicotomía identidad-alteridad (hombre-mujer) y sus consecuencias posmodernas para, así, profundizar en nuevos y terceros espacios, plenos de opciones que nos lleven a facultar intersticios, tendencias liminares donde prevalezca lo inter(entre)cultural. Habilitando, con esto, espacios intermedios, pensamientos del ENTRE (Derrida, 1967) que ayuden a estimular relaciones de tolerancia, con el fin de perfilar un nuevo emplazamiento -“(... ) algo nuevo...", nos sugiere Deleuze (1985, 1996: 240)- en el que exista una alianza en colaboración entre las diversas alternativas socio-culturales que circulan por nuestras sociedades (Cixous, 1995).

\section{REFERENCIAS BIBLIOGRÁFICAS}

Arriaga, M., Mi amor, mi juez, Alteridad autobiográfica femenina, Barcelona, Anthropos, 2001.

Bhabha, H. K., The location of culture, London and New York, Routledge,1994.

Browne, R., “Falogocentrismo: 'ella, es decir, lo otro'. Apuntes para una escritura matrística”, Actas del IV Seminario de la Asociación Universitaria de Estudio de Mujeres (AUDEM), en "Entretejiendo saberes". Sevilla, Universidad de Sevilla.

Cixous, H., "La joven nacida. II Salidas", La risa de la medusa. Ensayos sobre la escritura, Barcelona, Anthropos, 1995.

----, La risa de la medusa. Ensayos sobre la escritura, Barcelona, Anthropos, 1995.

Deleuze, G., Lógica del sentido, Barcelona, Paidós, 1989.

----, Deseo y Placer, Archipiélago, 23, Barcelona, 1995.
Deleuze, G. y Guattari, F., El AntiEdipo Capitalismo y esquizofrenia, Barcelona, Paidós, 1995.

Deleuze, G. y Parnet, C., Diálogos, Valencia, Pre-textos, 1997.

Derrida, J., De la Gramatología, Buenos Aires, Siglo XXI, 1971

Foucault, M., Las palabras y las cosas, México, Siglo XXI, 1986.

(1969): “Qué es un autor?”, Henciclopedia, Montevideo, http://www.henciplopedia. org.uy

Guattari, F., Caosmosis, Buenos Aires, Manantial, 1996.

Hardt, M. y N., A., Imperio, Buenos Aires, Paidós, 2002.

Ibañez, J., El regreso del sujeto. La investigación social de segundo orden, Madrid, Siglo XXI, 1994.

Rodrigo Alsina, M., "Apuntes sobre una semiótica intercultural", en Actas del lX Congreso de la Asociación Española de Semiótica "Humanidades, ciencia y tecnologías", Valencia, 2000.

Silva Echeto, V., Nuevas perspectivas teóricas de la comunicación. La dominación del Imperio, Paideia. Divulgación del pensamiento crítico, México, UAM, 2003a.

----, Comunicación e Información (inter)cultural, Sevilla, Instituto Europeo de Comunicación y Desarrollo, 2003b.

Vázquez Medel, M. Á., Mujer, ecología y comunicación en el nuevo horizonte planetario, Sevilla, Mergablum, 1999.

Vidal, R., “Discurso feminista y temporalidad: la descomposición postmoderna de las identidades de género", Actas del IX Simposio Internacional de la Asociación Andaluza de Semiótica "Mujer, Cultura y Comunicación: realidades e imaginarios", Sevilla, Alfar y Universidad de Sevilla, 2001 